\title{
Sperm cytology: Kernechtrot-picroindigocarmine stain and dog semen
}

\author{
Adam W Stern DVM DACVP1
}

\begin{abstract}
Animal sexual abuse cases present as an unusual scenario for forensic investigators. In this study, the kernechtrot-picroindigocarmine (KPIC, aka Christmas tree stain) staining properties of semen from 8 domestic dogs was compared to two samples of human sperm. In the head of the dog sperm, the acrosomal region stained pale red and the postacrosomal region stained dark red. There was a colorless band with a mean length of $0.59 \mu \mathrm{m}$ within the sperm head between the acrosomal and postacrosomal regions. The colorless band was not identified within the human control samples nor reported in the literature. This data supports the use of KPIC stain for the examination of dog semen and further characterizes a unique staining pattern found in dog sperm that is not present in human sperm.
\end{abstract}

KEYWORDS: veterinary forensic sciences, companion animals, sexual abuse, semen, dog, Christmas tree stain, kernechtrot-picroindigocarmine stain, KPIC

In cases of suspected human sexual assault, evaluation for semen is of high priority, and collection of trace evidence should be performed whenever a sex crime is suspected. Semen is composed of spermatozoa (cellular component) and seminal plasma (fluid component). There are several screening tests for semen available for use, including acid phosphatase tests, prostate-specific antigen (PSA) tests, semenogelin tests, and mass spectrometry-based proteomics (Hochmeister et al. 1999, Khaldi et al. 2004, Stern and Lanka 2016, Van Steendam et al. 2013). Sperm cytology can be used as a confirmatory test in forensic cases (Martinez et al. 2015). Additionally, there are other confirmatory tests available, including analysis of short tandem repeat loci from the $\mathrm{Y}$ chromosome (Y-STR) and mass spectrometry-based proteomics (Martinez et al. 2015, Van Steendam et al. 2013).

Sperm is composed of a head, midpiece, and tail. The oval head of human sperm measures an average of $4.6 \mu \mathrm{m}$ in length and $2.6 \mu \mathrm{m}$ in width (Schudel 2001). The midpiece contains numerous mitochondria. The tail is used for motility and is approximately $40 \mu \mathrm{m}$ in length. The tail is fragile and is easily detached from the head. When stained with KPIC stain, the head stains red and the acrosome, clear to pale red (Schudel 2001).

In cases of animal sexual abuse, sperm are sometimes recovered from the dog's fur or by vaginal or rectal washings or swabs. If sperm are recovered, it is imperative to determine whether the sperm is of human or animal origin. Although various commercially available tests can be used to evaluate human semen, these tests cannot readily identify the presence of canine semen. Using a commercially available membrane strip test, semenogelin, human semen has been shown to not crossreact with canine semen (Stern and Lanka 2016). Evaluation for PSA has resulted in conflicting results: one study documented canine semen as negative for PSA, another as positive for PSA (Hochmeister et al. 1999, Simich et al. 1999). Given the lack of adequate screening tests for canine semen, it is typical for the veterinary forensic investigator to initially perform a confirmatory test for semen, such as sperm cytology, to allow for visualization of sperm. For veterinary reproductive assessment, eosin-nigrosin and Wright-Giemsa stains are commonly used for semen evaluation (American Kennel Club Canine Health Foundation 2015). However, in the forensic context, KPIC stain should be used (Stern and Blackmore-Smith 2016).

The KPIC method is commonly used for the forensic evaluation of cytology slides for human sperm. It is simple to perform and commercially available. The kernechtrot stain (aka nuclear fast red) colors the sperm head red and is followed by the picroindigocarmine stain, which colors the sperm tail green; thus, the alternative name, Christmas tree stain. There is a single report in the literature describing the staining characteristics of sperm from a single dog when stained with KPIC. In this report, the sperm head is shown to contain a colorless band (Schudel 2001). The purpose of this study was to evaluate the staining characteristics of sperm from multiple dogs when stained with KPIC to serve as a reference for the examination of forensically relevant samples.

\section{Materials and Methods}

Raw semen from two different human subjects were used as controls. The samples used were medical waste. Institutional review board approval was obtained (University of Florida IRB protocol no. 20181040). Samples were stored at $2{ }^{\circ} \mathrm{C}$ for 48 hours. Direct aliquots of liquid semen were pipetted for analysis. The collection of dog semen was approved by the Institutional Animal Care and Use Committee (University of Illinois IACUC protocol no. 17283). Owner consent was obtained before the collection of the semen samples. Semen samples were collected from 8 healthy, male dogs by digital massage and pressure (Table 1). Samples

\footnotetext{
1 Department of Comparative, Diagnostic, and Population Medicine, University of Florida College of Veterinary Medicine, PO Box 100123, Gainesville, FL 32610-0123, USA 
were snap-frozen in liquid nitrogen and transferred to a $-80{ }^{\circ} \mathrm{C}$ freezer for long-term storage. Samples were thawed in a $37{ }^{\circ} \mathrm{C}$ water bath. Direct aliquots of liquid semen were pipetted for analysis.

TABLE 1-Signalment of dogs from which semen specimens were obtained for examination after application of

\begin{tabular}{lll}
\multicolumn{3}{c}{ kernechtrot-picroindigocarmine stain } \\
\hline Case & Breed & Age (years) \\
\hline 1 & Chihuahua & 8 \\
2 & Chihuahua & 7 \\
3 & Chihuahua & 3 \\
4 & Chihuahua & 3 \\
5 & Chihuahua & 6 \\
6 & German shepherd & 1.5 \\
7 & collie & 3 \\
8 & shih tzu & 6 \\
\hline
\end{tabular}

KPIC stain was used to identify sperm in the samples. Push smears of semen were prepared using $0.1 \mathrm{~mL}$ of semen. The samples were heat-fixed on a slide warmer (C\&A Scientific, Manassas, Virginia) at $65{ }^{\circ} \mathrm{C}$ for 20 minutes. The slides were then covered with nuclear fast red for 25 minutes and gently washed with $100 \%$ ethanol until the wash appeared clear. The slides were then counterstained with picroindigocarmine for 5 seconds and gently washed with deionized water.

Slides of semen were examined with a light microscope under $200 \times, 400 \times$, and $1000 \times$ oil emersion and photographed. Sperm were evaluated for staining characteristics.

Images of stained sperm were captured and digitized using cellSens Standard 1.17 software (Olympus Corporation, Tokyo, Japan) that was connected to a DP74 digital camera (Olympus Corporation, Tokyo, Japan). From the digitized images, various measurements were manually obtained using the measurement tools within the cellSens Standard 1.17 software. Twenty-five sperm were measured from each sample. Head length was measured as a linear length from the tip of the acrosome to the region where the head abuts the midpiece. The colorless band was measured from the acrosome-band junction to the bandpostacrosome junction. Additionally, the ratio between sperm head and colorless band (head:band) was calculated.

The null hypothesis is that the sizes of the sperm head and the colorless band were not different between study dogs, and was tested by using the one-sample $T$-test. Values of $P<0.05$ were considered significant. All calculations were performed using commercial software (Statistix 10, Tallahassee, Florida).

\section{Results}

Spermatozoa were observed in all human control specimens. Although most sperm were intact, individual sperm heads and tails were scattered within the slides. Overall, the sperm heads stained pale red, and the tails were green to gray (Figure 1). The acrosomal region of the sperm head stained pale red and the postacrosomal region was dark red.
Spermatozoa were observed in all dog specimens. Although most sperm were intact, individual sperm heads and tails were scattered within the slides. Overall, the sperm heads stained pale red, and the tails were green to gray. There was a small indentation in the head of the sperm where the midpiece abuts the head. The length of the sperm heads ranged in size from 4.58 to $7.74 \mu \mathrm{m}$; the mean length was $5.72 \mu \mathrm{m}$. The acrosomal region of the sperm head stains lighter than the posterior region. There was a colorless to occasionally very pale pink band separating the acrosomal region from the postacrosomal region (Figure 2). The length of the colorless band ranged from 0.37 to $0.88 \mu \mathrm{m}$; the mean length was $0.59 \mu \mathrm{m}$. The postacrosomal region stained darker red than the acrosomal cap. The mean ratio of the length of the sperm head to the length of the colorless band was 9.89 .

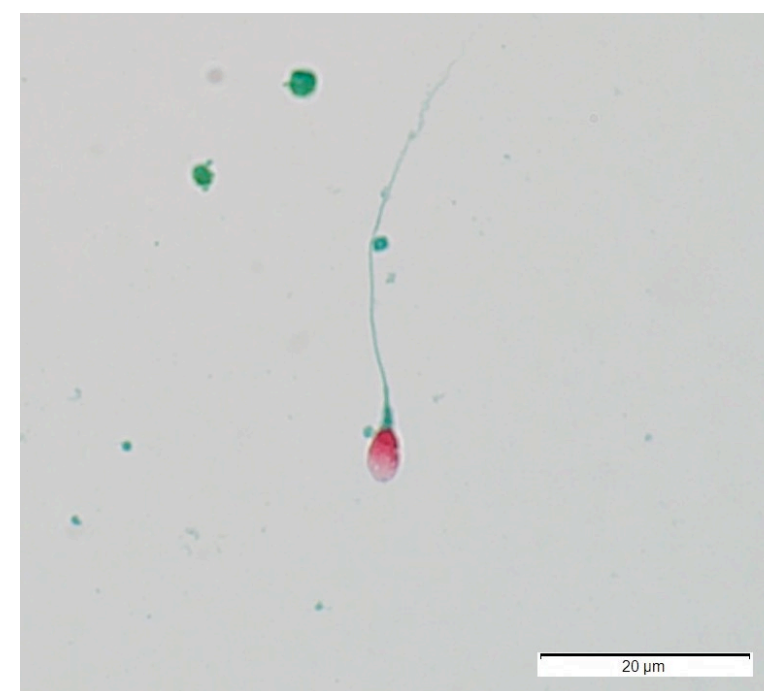

FIGURE 1-Cytology of human sperm Kernechtrot-picroindigocarmine stain, $1000 \times$ oil immersion.

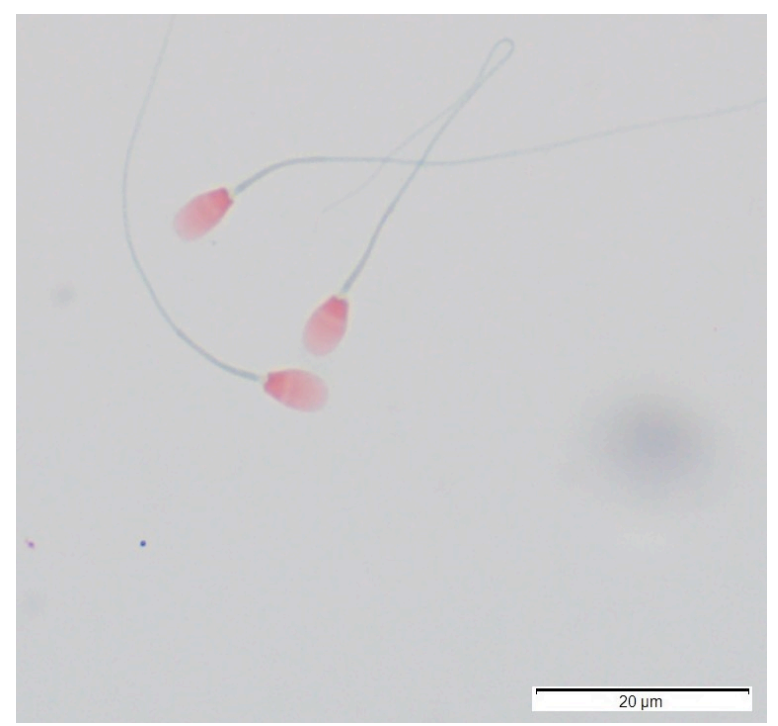

FIGURE 2-Cytology of dog sperm Note the colorless band within the sperm heads. Kernechtrot-picroindigocarmine stain, dog 3, 1000× oil immersion.

From data points from the five Chihuahua dogs, we can conclude that there is no difference in mean head length $(P=0.96$, 
mean $\pm S D=5.706 \pm 0.329 \mu \mathrm{m}$ ) and band length (mean $\pm S D=$ $0.589 \pm 0.017 \mu \mathrm{m})$. From data points from all eight dogs, we can conclude that there is no difference in mean head length $(P=1.0$, mean $\pm S D=5.72 \pm 0.27 \mu \mathrm{m})$ and band length $(P=1.0$, mean \pm $S D=0.59 \pm 0.018 \mu \mathrm{m})$ from the mean value of the Chihuahua dogs.

\section{Discussion}

Semen cytology is considered the gold standard for the detection of sperm in human sexual assault cases, although other methods that may be utilized include acquiring Y-STR profiles from samples that test positive for semenogelin (Martinez et al. 2015). Screening tests are useful for the detection of human semen; however, these tests have not been shown to be useful for the detection of dog semen. As a result, other methods such as sperm cytology should be used to evaluate for dog semen.

Similar to human sperm, it was shown that when KPIC is used to stain dog sperm, the acrosome is pale red and the postacrosomal region is red to dark red. In contrast to human sperm, sperm heads from all dogs had a colorless band separating the acrosomal and postacrosomal regions. There was no statistical difference between the mean head length and band length from all eight dogs from the mean value of the Chihuahua dogs in this study. This band has not been reported to be visualized with other stains such as Diff-Quik and Giemsa that are used to assess sperm head morphology from dog sperm (American Kennel Club Canine Health Foundation 2015, Soler et al. 2017, Dahlborn et al. 1996).

The results of this study further support the observation of a colorless band, which was identified in all dog semen specimens evaluated, that separated the acrosomal and postacrosomal regions of the dog sperm head. The human semen and dog semen were stored differently in this study, and it is plausible to hypothesize that the storage conditions could result in the formation of the band. The author has observed colorless bands in dog sperm found in urine and fresh dog semen (data not shown); thus, storage conditions do not appear to affect the appearance of the band within the sperm head of the dog. The finding of a colorless band within the sperm head is of interest to the forensic analyst as it characterizes a unique difference between dog sperm and human sperm based on cytological methods. If necessary, DNA testing of the sample can then be performed to verify the species of origin. It is possible to use laser capture microdissection to remove the sperm from the sample in order to obtain DNA profiles (Vandewoestyne and Deforce 2010).

\section{Conclusions}

In this study, semen from four breeds of dog were examined. Future studies using increased breed variation should be performed to confirm the colorless band feature in additional breeds. Evaluation of sperm from other domestic animal species should be performed in order to assess for the presence or absence of a colorless band within the sperm head. As not all forensic samples are in good condition, the effects of degradation of semen samples and the presence or absence of the colorless band should also be evaluated.
The data presented here confirm that the head of the dog sperm has an unusual staining pattern with a colorless band identified between the acrosomal and postacrosomal regions of the sperm head. This colorless band is not identified within human sperm heads. The findings support the use of the KPIC stain for the examination of dog semen and further characterizes a unique staining pattern found in dog sperm that is not present in human sperm. The described colorless band within the sperm head may be useful to differentiate between dog and human sperm; further studies about this morphologic feature are warranted.

\section{Acknowledgments}

The author would like to thank Thomas Denagamage for statistical assistance.

\section{Disclosures}

This research did not receive any specific grant from funding agencies in the public, commercial, or not-for-profit sectors.

\section{References}

American Kennel Club Canine Health Foundation. 2015. Canine semen evaluation. [Accessed 11 Jan 2019]. akcchf.org/educational-resources/podcasts/podcasttranscripts/Canine-Semen-Evaluation-Dr-Cheryl-Lopate.pdf

Dahlborn M, Andersson M, Vierula M, Alliance M. 1996. Morphometry of normal and tertazoospermic canine sperm heads using an image analyzer: work in progress. Theriogenology. 48:687-98.

Hochmeister MN, Boodle B, Rudin O, Gehrig C, Borer U, Thali M, Dirnhofer R. 1999. Evaluation of prostate-specific antigen (PSA) membrane test assays for the forensic evaluation of seminal fluid. J Forensic Sci. 44:1057-60.

Khaldi N, Miras A, Botti K, Gromb S. 2004. Evaluation of three rapid detection methods for the forensic identification of seminal fluid in rape cases. J Forensic Sci. 49:749-53.

Martinez P, Santiago B, Alcala B, Atienza I. 2015. Semen searching when sperm is absent. Sci Justice. 55:118-23.

Schudel D. 2001. Screening for canine spermatozoa. Sci Justice. 41:117-9.

Simich JP, Morris SL, Klick RL, Rittenhouse-Diakun K. 1999. Validation of the use of a commercially available kit for the identification of prostate specific antigen (PSA) in semen stains. J Forensic Sci. 44:1229-31.

Soler C, Alambiaga A, Martí MA, García-Molina A, Valverde A, Contell J, Campos M. 2017. Dog sperm head morphometry: its diversity and evolution. Asian J Androl. 19:149-53.

Stern AW, Lanka S. 2016. Evaluation of human semenogelin membrane strip test for species cross-reactivity in dogs. Vet Pathol. 53:1095-8.

Stern AW, Smith-Blackmore M. 2016. Veterinary forensic pathology of animal sexual abuse. Vet Pathol. 53:1057-66.

Vandewoestyne M, Deforce D. 2010. Laser capture microdissection in forensic research: a review. Int J Leg Med. 124:513-21.

van Steendam K, de Ceuleneer M, Dhaenens M, van Hoofstat D, Deforce D. 2013. Mass spectrometry-based proteomics as a tool to identify biological matrices in forensic science. Int J Leg Med. 127:287-98. 